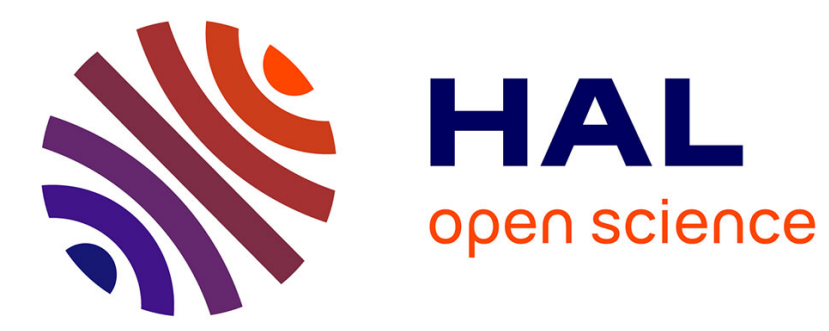

\title{
Prolongements du métropolitain et occupation du sol en banlieue parisienne
}

Padeiro Miguel

\section{To cite this version:}

Padeiro Miguel. Prolongements du métropolitain et occupation du sol en banlieue parisienne. Flux -

Cahiers scientifiques internationaux Réseaux et territoires, 2009, 1 (75), pp.42-56. halshs-00465534

\section{HAL Id: halshs-00465534 \\ https://shs.hal.science/halshs-00465534}

Submitted on 19 Mar 2010

HAL is a multi-disciplinary open access archive for the deposit and dissemination of scientific research documents, whether they are published or not. The documents may come from teaching and research institutions in France or abroad, or from public or private research centers.
L'archive ouverte pluridisciplinaire HAL, est destinée au dépôt et à la diffusion de documents scientifiques de niveau recherche, publiés ou non, émanant des établissements d'enseignement et de recherche français ou étrangers, des laboratoires publics ou privés. 


\title{
Le métro hors les murs : extension du réseau et occupation du sol en banlieue parisienne
}

\author{
Miguel Padeiro
}

Miguel Padeiro (Géographe, Doctorant en Urbanisme et Aménagement) Laboratoire Ville, Mobilité, Transports (INRETS-ENPC-Université Paris-Est) 19, rue Alfred Nobel - Cité Descartes - Champs-sur-Marne / F-77455 Marne la Vallée cedex 2

Telef. : +33164152128

Fax : +33164152140

E-mail : miguel.padeiro@enpc.fr

Financement ADEME - INRETS

Sous la direction de Francis Godard

Le développement du réseau métropolitain hors de son berceau, qui se réalise en continu depuis le début des années soixante-dix, met en jeu le rattachement fonctionnel à Paris d'un grand nombre de communes de la proche banlieue parisienne. Dans un contexte marqué par le double mouvement de globalisation/métropolisation, par l'accroissement des mobilités, notamment automobiles, et par l'émergence des problématiques sur le développement durable, les enjeux régionaux invitent à une plus grande maîtrise de l'occupation du sol dans le souci à la fois de rééquilibrages spatiaux au sein de la tache urbaine de l'agglomération et dans celui d'enrayer l'étalement qui se vérifie sur les marges. L'établissement de secteurs de densification préférentielle répond précisément à ce double objectif et apparaît clairement dans le projet du SDRIF (Schéma Directeur de la Région Île-de-France) arrêté par le Conseil Régional en février 2007, qui élit des périmètres de densification préférentielle dans un rayon de $2 \mathrm{~km}$ autour des gares et stations de banlieue.

L'idée n'est pas nouvelle de favoriser, en matière de densification et de polarisation urbaines, les axes du réseau lourd aux abords de la capitale. Le Schéma Directeur d'Aménagement et d'Urbanisme de la Région Parisienne (SDAURP), en 1965, comportait déjà de telles orientations. De nombreuses études menées par l'IAURIF (Institut d'Aménagement et d'Urbanisme de la Région Île-de-France), la DREIF (Direction Régionale de l'Équipement d'Île-de-France) et la RATP ont longtemps porté, depuis les années soixante-dix, sur ce thème de la valorisation de l'environnement des dessertes. Il est cependant permis de se demander, aujourd'hui, dans quelle mesure l'injonction des pouvoirs publics à la densification privilégiée sur certains axes et pôles, s'appuyant sur les infrastructures de transports, a été suivie d'effets. On sait que le SDAURP, par exemple, a souffert du poids conséquent d'urbanisations concurrentes (Steinberg, 1981, cité par Larroque, Margairaz, Zembri, 2002 : 269)

Dans ce but, nous replaçons dans un premier temps l'extension du réseau métropolitain, depuis les années soixante-dix, dans ses contextes successifs que marquent deux grandes orientations urbanistiques à l'échelle régionale (1). Dans un deuxième temps, nous exposons quelques résultats issus de l'exploitation de la base de données du Mode d'Occupation du Sol (MOS), fournie par l'IAURIF, concentrés sur quelques thématiques principales aptes à témoigner de différentiels spatiaux dans l'usage du sol (2). Nous tirons enfin quelques conclusions et discutons l'intérêt de mesures prescriptives et intégratrices liées à l'élaboration des projets d'infrastructures de transports (3).

\section{Prolongements de lignes, polarisation spatiale?}

Lorsque roula, en juillet 1900, la première rame du métro parisien, il ne faisait de doute pour personne que le nouveau réseau franchirait rapidement les limites de la capitale. Objet, à l'origine, d'âpres discussions entre une 
Assemblée Municipale de Paris radicale et autonomiste qui souhaitait donner au métro une fonction strictement locale, un État dont l'ambition était de conserver son pouvoir sur le territoire parisien et une nébuleuse de compagnies de chemin de fer qui craignaient de perdre d'importantes parts de marché, le métro s'affirma définitivement, au cours du long processus de sa création, comme la «chose de la municipalité » (Larroque, Margairaz, Zembri, 2002) et devait même par la suite s'ériger en symbole de la capitale. Robert Buron, Ministre des Travaux Publics, déclarant en 1961 que «le métro, c'est Paris », ne révèlera-t-il pas cette appropriation symbolique et politique du métro par les édiles locaux et par les habitants, cette reconnaissance par l'État d'une réalité devenue permanente? Pour autant, devant la croissance de l'agglomération parisienne, l'intensification des migrations alternantes entre centre et périphérie et l'augmentation de la demande de transports, les projets de prolongements de lignes ne tarderaient guère à essaimer. Contribuant à rendre criante la dissonance entre un dedans et un dehors, ces projets devaient souligner publiquement l'inadéquation du réseau existant et la solidarité fonctionnelle de deux espaces mal séparés par la ceinture des Habitations à Bon Marché (H.B.M.) que viendrait plus tard compléter le Boulevard Périphérique.

Justifiées par plusieurs rapports publics et plans d'aménagement qui devaient aboutir en 1934 au Plan Prost, vingt-six stations hors les murs surgirent ainsi entre 1934 et 1952, précédant une phase durable de faibles investissements et rares décisions en matière de transport public (Merlin, 1997 ; Larroque, Margairaz, Zembri, 2002), essentiellement marquée par les besoins de la reconstruction d'après-guerre, par la croissance urbaine et par la progressive diffusion de l'automobile au sein de la société française. À la fin des années soixante, dans un contexte favorable à une forte implication étatique dans les questions urbaines, une nouvelle période devait débuter, quasiment ininterrompue jusqu' aujourd'hui. Les vingt-huit stations suburbaines mises en service au cours de cette nouvelle phase d'expansion représentent aujourd'hui $60 \%$ des actuels cinquante-cinq kilomètres de lignes de ce métro extra-muros (Fig. I).

Fig. I - croissance cumulative du réseau métropolitain en banlieue parisienne (1934-2007)

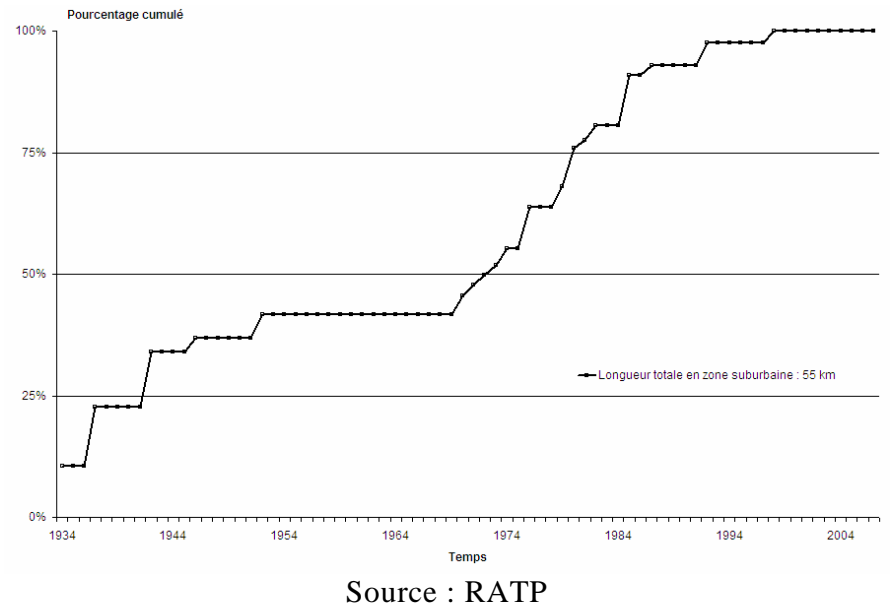

L'expansion du métro en banlieue parisienne doit être mise en relation avec deux grandes orientations urbanistiques qui scandèrent, depuis la Deuxième Guerre Mondiale, les politiques régionales régulièrement décrites comme un constant va-et-vient d'hésitations entre les tentations du malthusianisme polycentrique et le renforcement de la puissance et de la centralité parisiennes (Cornu, 1972 ; Fourcaut, Flonneau, 2005). Les premières se basent sur les thèses graviéristes, aux yeux desquelles le poids de Paris, aux échelles régionale et nationale, constituait un danger économique et social. Les premières orientations urbanistiques et économiques eurent ainsi pour principal objectif de diminuer ou, tout au moins, de stabiliser la population et l'emploi dans le centre d'une région capitale en plein développement. C'est dans ce contexte, commente (Cornu, 1972), que furent refusées plus de 300 demandes d'autorisation d'implantations industrielles en petite couronne. Accompagné et confirmé par une stratégie institutionnelle consciente et délibérée, le processus de désindustrialisation ne se révéla pas comme une simple résultante d'évolutions économiques, fussent-elles de longue portée spatiale et temporelle. À l'échelle urbaine, six centres administratifs et économiques, dits restructurateurs, furent ainsi définis, dont les 
principaux, à savoir Créteil, Bobigny et Nanterre, devaient désormais être rapidement reliés à Paris, les deux premiers étant finalement atteints par le métro respectivement en 1974 et en 1985. Il ne s'est pas agi, dans de tels cas, de répondre à une demande sociale existant lors de la mise sur agenda des projets en question. Ceux-ci, du reste, n'apparaissaient pas même parmi les opérations désignées comme prioritaires dans les plans quinquennaux de la RATP au cours des années cinquante et 1960 : il est vrai que la récurrente mise sur l'agenda politique du développement futur du RER eut probablement un effet de blocage sur les développements du métro (Larroque, Margairaz, Zembri, 2002). Ceux-ci devaient finalement s'avérer primordiaux dans les documents stratégiques aux niveaux régional et national, à l'instar de la révision du SDAURP, en 1969, ou du VI ${ }^{\mathrm{e}}$ Plan de Modernisation et d'Équipement pour la période 1971-1975, publié en 1971. Aussi peut-on lire, dans le préambule du schéma de principe du prolongement de la ligne 5 à Bobigny qui doit traverser des quartiers socialement marqués, des friches industrielles et des emprises ferroviaires, que « les population et emplois que l'on y rencontre n'auraient pas justifié à eux seuls (l'élaboration du projet) si, après la réforme administrative de 1964 de la région parisienne, il n'avait pas été décidé de faire de Bobigny (...) le chef-lieu du nouveau département de la Seine-Saint-Denis » (RATP, 1979b : 5). Ces prolongements s'inscrivent dans des projets de grande envergure qui ont obligé à une temporalité rigoureuse, accéléré la période entre projet et mise en service : les prolongements de Créteil (ligne 8, 1974), de Bobigny (ligne 5, 1985), de La Défense (ligne 1, 1992) et de l'Université de Saint-Denis (ligne 13, 1998) appartiennent à cette catégories d'opérations prioritaires concrétisées dans un délai de dix à quinze ans et qui ont trouvé leur justification dans les politiques de décentralisation administrative et économique. Ces dernières avaient également impulsé, dès le début des années soixante, la création de lignes RER censées constituer une ossature de premier rang à l'échelle régionale ${ }^{1}$.

Les secondes grandes orientations, moins volontaristes en matière d'aménagement du territoire, tentèrent de multiplier les liaisons entre Paris et ses environs dans le but de résoudre les problèmes nés de l'important accroissement des migrations pendulaires radiales et de la congestion automobile, facteurs de paralysie des grandes voies d'accès à Paris. Ce groupe de prolongements de lignes répondait à une demande sociale de plus en plus intense et à un accroissement démographique qui atteignait toutes les communes limitrophes de Paris, les nouvelles lignes s'inscrivant dans un tissu à prépondérance résidentielle qui conférait aux projets une logique justification, insuffisante néanmoins pour imposer automatiquement un calendrier d'urgence lors de l'élaboration des projets. C'est ainsi que plusieurs stations apparaissent avec plusieurs décennies de retard par rapport aux projets initiaux. Le prolongement à Clichy de la ligne 13 est exemplaire à ce titre : inauguré en 1980, il avait été déclaré d'utilité publique le $1^{\mathrm{er}}$ janvier $1930^{2}$. Le prolongement de la ligne 4 au Sud de Paris, vers Bagneux et Montrouge, proposé dès 1919, déclaré d'utilité publique en 1930, est aujourd'hui en cours de réalisation, la station Mairie de Montrouge devant ouvrir en 2011 en un lieu différent de celui envisagé durant la première moitié du XXe siècle. Une demande sociale préexistante, qu'accompagnent d'incessantes revendications locales, participe donc de la lente émergence des stations du réseau suburbain, avec une temporalité différente.

Fig. II - Lignes prolongées en banlieue parisienne

\footnotetext{
${ }^{1}$ Nos travaux portant sur les prolongements du métro parisien en banlieue, nous ne développerons pas ici la question du RER qui constitue un réseau géographiquement et fonctionnellement distinct d'un réseau métropolitain dont la desserte fine est la caractéristique principale.

${ }^{2}$ Le même destin caractérise l'autre branche de la ligne 13, vers Saint-Denis. Déclaré d'utilité publique en décembre 1929, son exécution fut prévue par la loi du 04 juin 1941. Entre 1951 et 1964, pas moins de 15 avis favorables à l'unanimité du Conseil Général de la Seine et 25 délibérations du Conseil Municipal de Saint-Denis ont été émis dans le but d'obtenir le prolongement (Archives STP 581 WR 257). Il faudra pourtant attendre 1976 pour que le centre-ville soit desservi.
} 


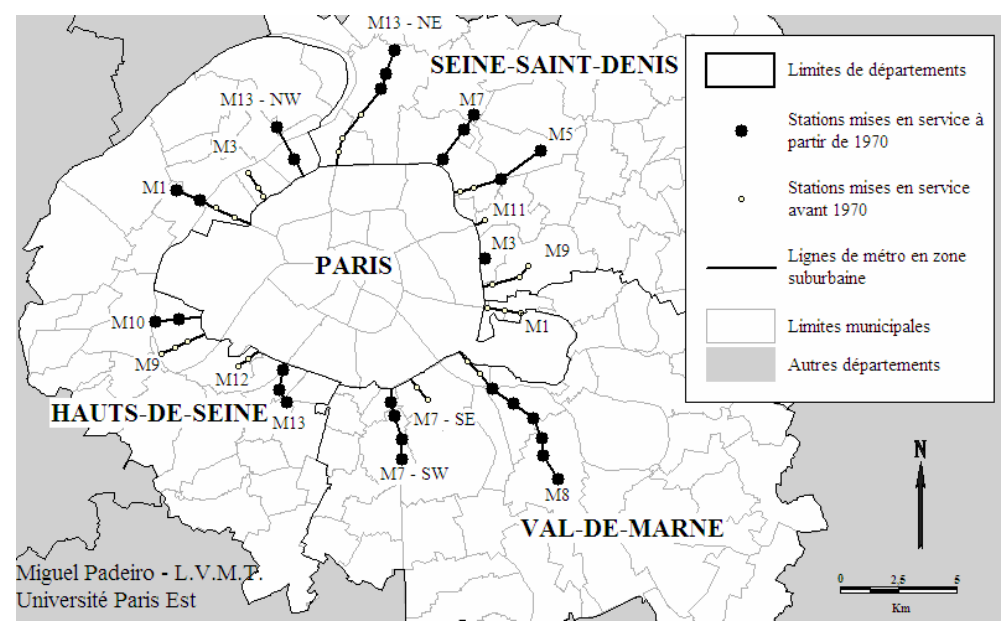

Le réseau métropolitain, dans sa partie suburbaine, illustre donc les deux versants de la croissance de l'agglomération parisienne (Fig. II) : centralité ou expansion périphérique, confirmation de l'accumulation sur quelques axes privilégiés de l'espace bâti au long du temps, fragiles tentatives de modeler l'ensemble urbain après la Seconde Guerre Mondiale. Par des voies différentes, les deux ensembles aboutissent à une mosaïque linéaire incomplète dont le caractère exclusivement radial est sous-jacent à toutes les orientations définies jusqu'à présent. Ces dernières, fondées en outre sur les revendications des édiles municipaux qui y gagnent, selon Jacques Gozard (Délégué Général du GIE Ville et Transports), une honorabilité symbolique et politique ${ }^{3}$, obtiennent généralement le soutien de populations soucieuses d'une plus grande aisance dans leurs déplacements pendulaires vers le premier pôle culturel et économique de la région. D'une haute densité dans le noyau central, fort de ses 243 stations disséminées sur un territoire de $105 \mathrm{~km}^{2}$, le réseau se fait plus épars hors des limites administratives de Paris, et les interconnexions avec d'autres réseaux deviennent quasi-inexistantes : une seule correspondance existe entre métro et RER (station La Défense Grande Arche), une autre entre métro et ligne de tramway (station Bobigny Pablo Picasso), mais aucune connexion métro-métro ne vient à ce jour compléter la gamme de trajets possibles. Le cloisonnement est une réalité du métro suburbain, non dissociable de sa dépendance historique, géographique et fonctionnelle vis-à-vis de l'aire centrale parisienne.

Cette subordination d'un réseau lourd à son noyau central guide dès lors la réflexion vers le maintien d'une centralité parisienne élargie à la banlieue proche, en contrepoint des théories et des discours sur le polycentrisme et l'extension des marges urbaines. L'importance d'une localisation centrale n'est pas annihilée par les modes de transports et de communication (Carroué, 2000 ; Cohen, 2002 ; Burgel, 1999) et maints auteurs ont décrit en détail les mouvements de reconquête des espaces centraux par certaines couches de la population (Smith, 1999 ; Butler, 1997 ; Mendes, 2006) ou par les acteurs économiques (Beckouche, Vire, 1998), illustrant ainsi une évolution dont l'image, dans le cas francilien, «atténue, sinon révoque, la croyance tenace en une polycentralité parisienne » (Burgel, 2006). L'accessibilité étant définie par «l'aptitude à la centralité d'un territoire » (Wenglenski, 2003), nous émettons l'hypothèse d'une différenciation spatiale qu'entraînerait l'apparition d'une station de métro, indépendamment du processus socio-historique et politique qui a provoqué son apparition. L'accessibilité plus rapide à une ville centre qui se maintient, malgré le processus d'extension périphérique, comme le premier pôle d'emploi de la région (Burgel, 1999), est supposé entraîner, dans un contexte caractérisé par la logique de compétition entre acteurs, ménages et entreprises, une augmentation de l'urbanité qui, définie «comme situation productive, consiste en la mise en co-présence du maximum d'objets sociaux dans une conjonction de distances minimales » (Lévy, 1994). Les alentours de la station de métro insérée en un tissu hétérogène, à l'urbanité incomplète - la banlieue comme «anneau de tôles et de presses » (Carroué, 2000) - serait alors l'objet d'une densification privilégiée, sélective et progressive, par intensification des surfaces bâties et des activités. Notre hypothèse de travail peut alors se formuler de la manière suivante : la construction d'une station, comme point

\footnotetext{
${ }^{3}$ Communication personnelle.
} 
d'entrée de la banlieue vers l'aire centrale de l'agglomération urbaine, crée les conditions de différenciation spatiale nécessaires pour se réfléchir, à proximité (dimension spatiale) et à moyen terme (dimension temporelle), sur l'intensité de l'usage économique et résidentiel du sol, et sur la mise en valeur de l'espace libre ou vacant.

Il s'agit donc de répondre à deux grandes questions que continuent de poser les acteurs de l'aménagement et de l'urbanisme : y a-t-il eu une plus grande valorisation ${ }^{4}$ de l'espace libre situé à proximité des stations de métro par rapport à des secteurs plus éloignés ? Existe-t-il une polarisation ${ }^{5}$ de l'usage du sol autour de ces mêmes stations, en termes résidentiels ou d'activités, autrement dit se produit-il une attraction particulière touchant les occupations moins consommatrices de sol urbain, par opposition aux plus extensives? Analysant l'évolution de l'occupation du sol autour de huit stations de métro et onze gares de banlieue, les auteurs d'un rapport publié par l'IAURIF ont détecté une tendance au remplacement de surfaces de résidence individuelle par des surfaces d'habitations collectives au cours de la période 1982-2004; les activités auraient suivi une trajectoire similaire, les plus extensives cédant du terrain aux plus intensives (Said, Santoire, Hengoat, 2001). Bien que non systématisés à l'échelle régionale, ces résultats rendent compte d'un ample mouvement de densification autour des stations de métro et des trains de banlieue, évoquant par ailleurs une possible valorisation de l'espace, une position qui s'inscrit dans le contexte actuel favorable à la diminution ou à la rationalisation de la consommation du sol urbain. D'autres analyses conduites sur le territoire de Montreuil, que le métro a atteint au cours des années trente, montrent en revanche une faible relation entre réseau métropolitain et occupation du sol ou rénovation du parc immobilier (Rajchman, 1980). L'auteur affirme que l'influence du métro se fait probablement sentir, en matière foncière et immobilière, sur le prix des terrains et des logements. Mais cette étude, au contraire de celle conduite par l'IAURIF, a été effectuée à l'échelle municipale: ce choix ou cette contrainte technico-méthodologique se répercute probablement sur les résultats obtenus.

\section{Une faible intensification de l'usage du sol}

Dans le but de détecter l'existence ou non d'un intensification sélective du tissu construit sur une période relativement longue, nous observons l'évolution de l'occupation du sol autour des 28 stations de métro construites depuis 1970. L'analyse porte ici sur trois thèmes qui constituent le cœur de la réflexion : activités économiques, tissu résidentiel et dynamique des espaces vacants sont tour à tour abordés, les deux premiers items en raison de leur importance en tant que générateurs de déplacements, le dernier parce qu'il représente un champ opérationnel concret dans les politiques d'aménagement et d'urbanisme, en particulier dans le cas d'une couronne suburbaine dont les potentialités foncières s'amenuisent avec le temps.

Encadré 1 : exploitation de la base de données MOS (IAURIF)

La base de données du MOS (Mode d'Occupation du Sol) constitue un « atlas cartographique informatisé de l'occupation du sol de la Région Île-de-France » (IAURIF, 2001). Élaborée par l'IAURIF depuis 1982 sur la base d'une couverture intégrale de la région parisienne par la photographie aérienne, elle a été plusieurs fois actualisée (1987, 1990, 1994, 1999 et 2003) et permet, à travers une nomenclature stable dans le temps, des comparaisons longitudinales. Cinq niveaux d'agrégation des données sont disponibles, les 3 modes (rural, urbain ouvert, urbain construit), se divisant en 11, 21, 48 puis 83 modes d'occupation différents.

\footnotetext{
${ }^{4}$ Le terme « valorisation » présente une double acception, d'accroissement de la valeur marchande d'une part, de mise en valeur par l'usage, éventuellement dans le but d'en tirer des ressources d'autre part. C'est dans ce dernier sens que nous l'utilisons.

${ }^{5}$ Par «polarisation de l'usage du sol », nous entendons toute tendance à l'augmentation de la part, en espace occupé, des usages les plus intensifs, c'est-à-dire mobilisant plus de personnes (emplois, résidents) sur moins de surface, par opposition à des usages extensifs, qui mobilisent plus d'espace et moins de personnes. A ce titre, une zone pavillonnaire est considérée comme plus extensive qu'une zone d'immeubles collectifs; des tours de bureaux font du sol un usage plus intensif qu'une zone d'activité industrielle.
} 
Chaque unité géographique est définie par trois caractéristiques distinctes : son évolution chronologique, son insertion dans un territoire municipal donné, sa continuité spatiale.

L'analyse des environs des vingt-huit stations étudiées a donné lieu à trois moments de choix méthodologiques. D'abord ont été construites, par la méthode cartographique des tampons (buffers), dix bandes concentriques de cent mètres de large, jusqu'à mille mètres, autour de chaque station. Cette technique ajoute, aux trois composantes de base des unités géographiques, une quatrième caractéristique : l'appartenance à un anneau concentrique numéroté. Le traitement appelle deux remarques : d'abord, nous avons procédé à deux modes d'agrégation des données : d'une part, nous avons groupé l'ensemble des stations en une seule et même station théorique, de manière à réaliser des observations au niveau régional ; d'autre part, nous avons agrégé les données par extrémités de lignes. Nous ne présentons, dans ce texte, que la première partie.

Les catégories de description forment le deuxième temps de définition des choix méthodologiques. Trois thèmes ont été abordés : les deux premiers sont classiques dans les rapports déjà publiés sur la base du MOS : il s'agit des activités économiques et des fonctions résidentielles, dont l'étude se justifie à la fois par leur poids dans l'espace (plus de $55 \%$ des environs des stations concernées) et par leur caractère central dans les politiques de maîtrise du sol à l'échelle régionale, en tant que composante fondamentale de la densification souhaitée dans les discours et les documents stratégiques que constituent le SDRIF de 1994 et de 2007. La dernière version du SDRIF, dont le projet a été arrêté en février 2007 par le Conseil Régional, a choisi des zones de densification dite «préférentielle» dans des secteurs les mieux desservis par les infrastructures de transport collectif. Les composantes principales de cette densification consistent en l'apparition de générateurs de déplacements, à savoir la construction de logements et de bureaux (SDRIF, 2007 ; Fouchier, 2007). Le troisième thème choisi est plus original : l'espace classé comme «vacant » n'apparaît que rarement dans les études habituelles et, quand il est analysé, l'est dans une perspective statique de potentialité qui ne correspond guère aux évolutions dynamiques et éventuellement contradictoires. Si l'extension globale des espace vacants a diminué de $29 \%$ en petite couronne entre 1982 et 2003, il n'en demeure pas moins que $61 \%$ des surfaces constatées en 2003 n'étaient pas classées «espaces vacants » en 1982. Autrement dit, non seulement l'instabilité des espaces vacants en constitue l'une des marques principales, mais l'apparition de nouveaux espaces vacants peut encore constituer une proportion considérable de l'espace.

La méthode d'analyse est le dernier moment de choix. La construction de bandes concentriques a pour objectif de construire des gradients d'intensité de l'usage du sol par des modes d'occupation donnés. Cette méthode a été utilisée à plusieurs reprises à l'échelle régionale pour mesurer des densités de population (Bonnafous, Tabourin, 1998 ; Fouchier, 2003), mais n'a pas été appliquée à l'échelle des stations de métro, la préférence allant généralement à une définition globale d'un secteur de 200 ha autour des stations (Said, Santoire, Hengoat, 2001). La méthode se justifie par l'hypothèse de polarisation graduelle de l'espace urbain à une échelle différente de celles habituellement utilisées. Il s'agit de mesurer la totalité de l'espace occupé par un certain type d'utilisation du sol, de calculer sa proportion dans l'anneau respectif puis de comparer les proportions obtenues pour chaque anneau. La même opération, effectuée pour deux dates différentes (1982 et 2003), permet une analyse synchronique. Dans le cas des espaces vacants, une méthode différente a été appliquée, que nous expliciterons dans le texte.

\section{Une faible évolution de l'occupation du sol par l'activité économique}

Utilisant une distinction fournie par la base de données entre secteurs secondaire et tertiaire, une première analyse de l'usage du sol par les activités économiques met en évidence deux tendances contraires (Fig. III). D'une part, le secteur tertiaire devient plus présent en proportion d'occupation du sol à proximité du métro qu'à une plus grande distance de celui-ci : c'est à partir de 500 mètres que les proportions deviennent indépendantes de la localisation relative. D'autre part, les activités du secteur secondaire présentent une distribution graduelle inversée par rapport aux activités tertiaires, la proportion augmentant avec l'éloignement au métro. Cette augmentation n'est pas linéaire puisque le secteur occupe presque $15 \%$ de l'espace situé à moins de 100 mètres d'une station, une donnée qui traduit bien le rôle historique qu'ont joué les grandes voies routières dans les choix de localisation industrielle. En évolution 1982-2003, le secteur tertiaire présente un accroissement général de l'occupation du sol, très légèrement supérieur aux abords du métro, alors que diminue, tout aussi globalement, la part des surfaces consacrées aux activités industrielles.

Fig. III - occupation de l'espace autour des stations de métro, selon la distance, par les secteurs industriel et tertiaire. 


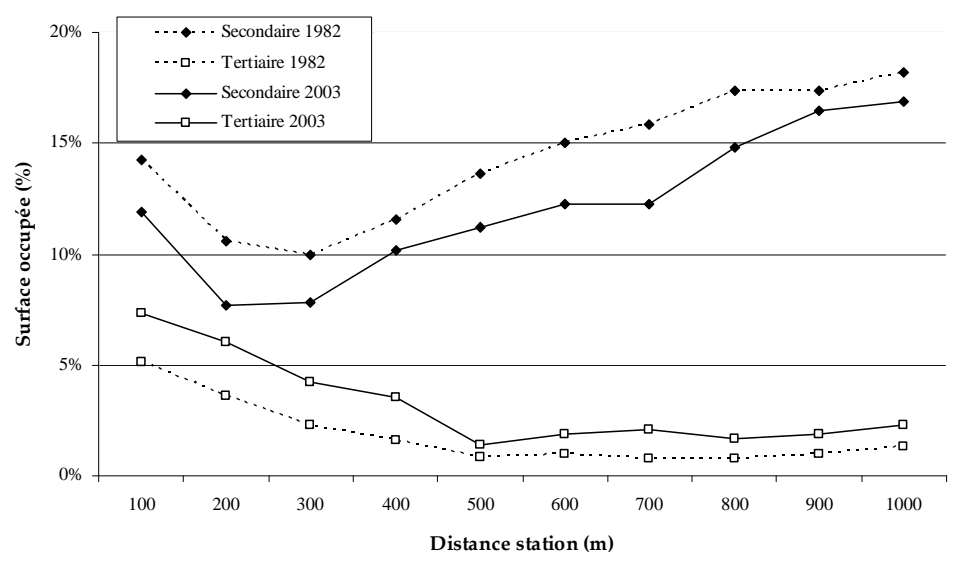

Source : IAURIF/MOS

Nous décomposons dans un deuxième temps les deux secteurs d'activités sur la base des classifications que le MOS permet d'opérer. Éliminant les surfaces de production animale, inexistantes en termes d'emprise au sol, les surfaces dédiées aux activités se rattachent à six modes différents (Tableau I). L'entreposage à l'air libre ne nous intéresse pas non plus : s'il ne représentait en 1999 pas moins de 12,8\% de la surface régionale consacrée aux activités, il ne constituait en revanche que $1 \%$ de l'espace desservi par le métro (INSEE, IAURIF, 2003 : 46). Dans ce dernier cas, l'espace consommé par chaque unité d'entreposage n'est guère compatible avec la rareté de l'espace disponible au cœur de l'agglomération urbaine; la répartition des rares unités dans l'espace central souligne du reste sa dépendance aux zones portuaires (Gennevilliers, Bonneuil-sur-Marne, canaux de Saint-Denis) et industrielles. L'analyse synchronique des autres types d'utilisation du sol mentionnés dans le Tableau I confirme, via l'existence de deux formes de distribution graduelle, les observations relatives aux activités secondaires et tertiaires. Les surfaces industrielles et les zones ou lotissements d'activités (Fig. IV), plus consommatrices d'espace, représentent $0,4 \%$ de l'espace situé à moins de 100 mètres d'une station, totalisant 0,38 ha. Leur présence augmente avec l'éloignement au métro, atteignant respectivement 6,9\% et 3,5\% dans l'anneau $900-1000$ mètres. Dans un deuxième groupe se trouvent les surfaces commerciales, les bureaux et les activités en tissu urbain mixte, ces dernières se définissant de la manière suivante : de caractère industriel, en locaux d'activités, laboratoires, entrepôts, ateliers qui, dispersés au sein de tissus à caractère principalement résidentiel, s'en distinguent néanmoins. Ce groupe obéit, au contraire du premier, à une répartition centripète (Fig. V). Tout en présentant des valeurs globalement faibles, le poids des surfaces commerciales augmente à proximité du métro, de même que les bureaux et les activités en tissu urbain mixte.

Tab. I - activités économiques présentes dans un rayon de 1000 mètres autour du métro suburbain de Paris, en 2003.

Modes d'occupation du sol (niveau d'agrégation : 48 modes)

\section{Surface occupée à moins de 1000 m d'une station de métro, en 2003 (ha)}

Activités en tissu urbain mixte

Grandes surfaces industrielles

Zones/lotissements d'activités

Entreposage à l'air libre

Surfaces commerciales

Bureaux

TOTAL

$\begin{array}{cc}264 & 33,4 \% \\ 227 & 28,8 \% \\ 135 & 17,1 \% \\ 33 & 4,2 \% \\ 44 & 5,6 \% \\ 85 & 10,8 \% \\ \mathbf{7 9 0} & \mathbf{1 0 0} \%\end{array}$

Pourcentage de la surface consacrée aux activités économiques, incluse dans un rayon de $1000 \mathrm{~m}$, en 2003

Source : IAURIF/MOS

Entre 1982 et 2003, l'écroulement des surfaces industrielles, encore plus perceptible à distance du réseau métropolitain, est quasi-total à proximité immédiate. Au cours de la même période, les zones et lotissements d'activités se sont accrus au-delà de 400 mètres, remplaçant en partie les surfaces industrielles (41,2\% de l'espace 
concerné était ainsi classé en 1982). Quant aux surfaces commerciales, elles ont augmenté de manière générale, indépendamment de leur position par rapport au métro.

Fig. IV - espace occupé par l'activité économique traditionnellement plus consommatrice d'espace, selon la distance au métro (1982-2003).
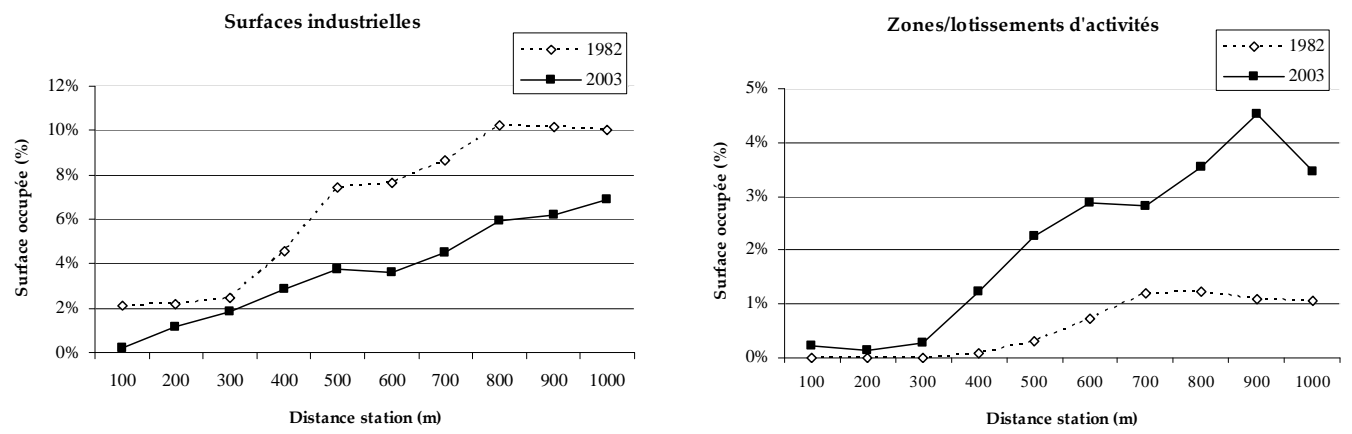

Souce : IAURIF/MOS

La forte croissance des surfaces de bureaux constatée au voisinage du métro (Fig. V), en particulier entre 200 et 400 mètres, tient pour partie au développement de La Défense. Le prolongement de la ligne 1 jusqu'à La Défense Grande Arche est lié à une Opération d'Intérêt National (OIN) portée par l'État depuis la fin des années cinquante, sur une aire qui compte aujourd'hui 150000 emplois et 20000 habitants. Ainsi, sur cette bande 200-400 m, La Défense n'accapare pas moins de $73 \%$ de la croissance des surfaces ; au-delà de la limite de $400 \mathrm{~m}$, la proportion tombe à $24 \%$. L'emprise au sol des bureaux a ainsi été multipliée par 2,4 au cours de la période.

Si nous excluons maintenant ce pôle économique majeur de l'analyse, et si nous nous intéressons toujours à cette bande 200-400 m, nous observons que les surfaces se sont multipliées par 2,9, passant de 2,2 à 6,4 ha; au-delà de $400 \mathrm{~m}$, les surfaces se sont multipliées par 3,12. Il s'est donc produit, certes, une augmentation des surfaces de bureaux aux abords du métro mais, sans La Défense, la même augmentation serait encore plus remarquable en des localisations plus éloignées que dans les environs du réseau.

L'occupation du sol par les activités en tissu urbain mixte a, quant à elle, légèrement diminué au cours de cette période, de manière plus marquée entre 100 et 400 mètres : sur $40 \%$ des 23,5 ha transformés, de nouvelles zones résidentielles sont apparues, auxquelles se sont ajoutées des bureaux (15\%).

Il n'est pas possible d'affirmer, au vu de ces données, qu'une évolution ample ou brutale se soit produite au cours de cette période de temps relativement large. La structure qui se vérifie en 2003 existait déjà en 1982 et n’a subi que quelques retouches : la multiplication des zones de bureaux n'est guère spécifique, en-dehors de La Défense, à la proximité du métro.

Fig. V - espace occupé par l'activité économique traditionnellement moins consommatrice d'espace, selon la distance au métro (19822003). 

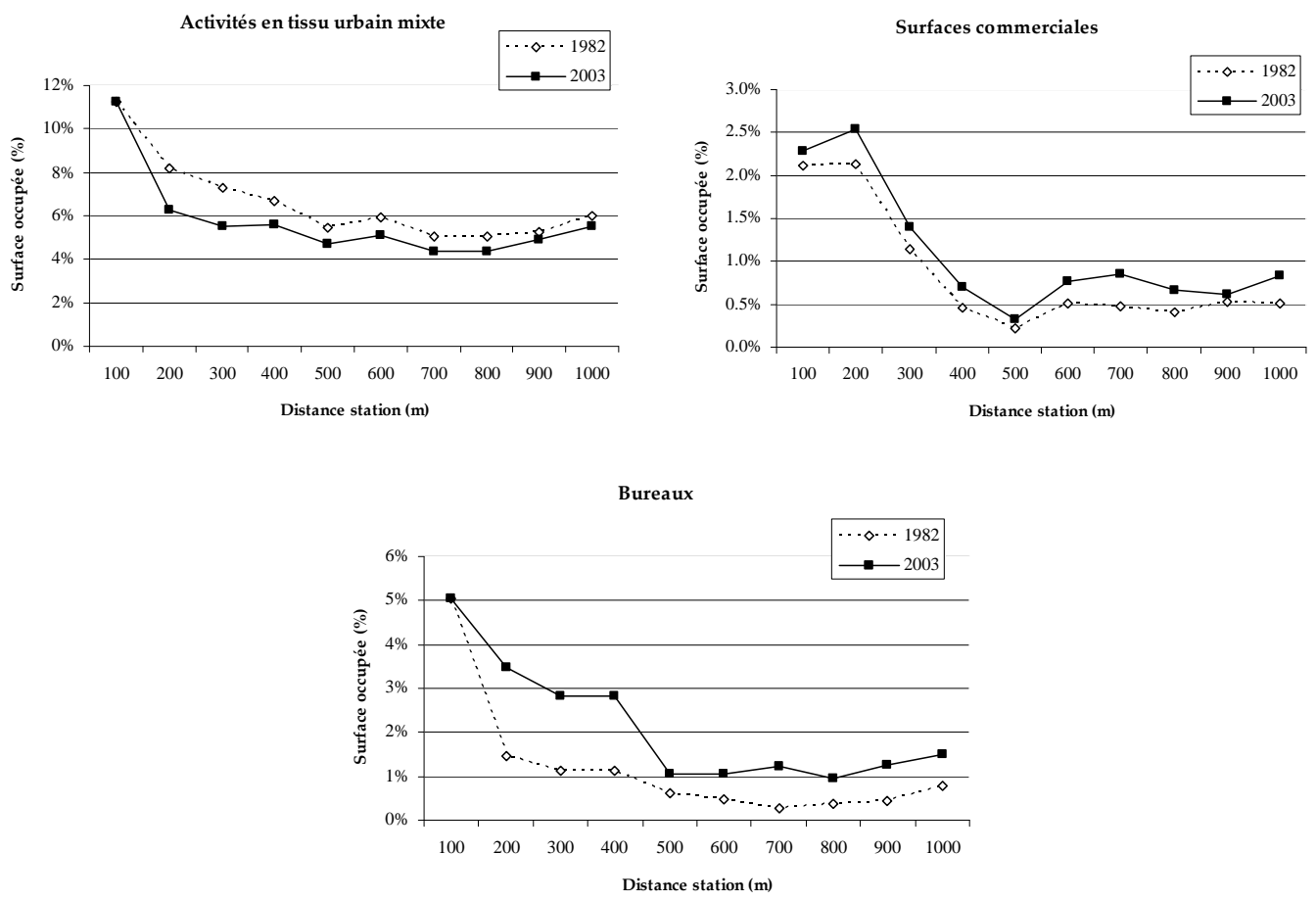

Source : IAURIF/MOS

\section{L'intensification relative des fonctions résidentielles}

Comme pour les activités économiques, deux grands types d'aires résidentielles ont été distingués dans un premier temps, qui correspondent à la classification de la base de données en onze modes d'occupation du sol : zones d'habitat individuel et zones d'habitat collectif. Les premières, qui réunissent $13 \%$ de l'espace considéré, regroupent toutes les constructions individuelles, éventuellement en urbanisations récentes (lotissements). Les secondes, représentant quant à elles $25 \%$ de l'espace, regroupent toutes les constructions d'habitat collectif. La stabilité, entre 1982 et 2003, est le principal trait de ces zones dont seulement $2 \%$ ont changé d'affectation entre les deux dates.

Là encore, à l'instar des activités économiques, deux tendances s'individualisent nettement (Fig. VI). D'une part, la proportion des surfaces résidentielles collectives croît graduellement à mesure que décroît la distance au réseau métropolitain : si $18 \%$ de l'espace au-delà de la limite de mille mètres est ainsi classé, la proportion passe à $22 \%$ dans l'anneau 900 - 1000 mètres, pour atteindre $30 \%$ dans les 200 mètres. C'est en sens inverse que se répartissent les surfaces d'habitation individuelle, qui augmentent de 6 à $16 \%$ entre le premier et le dernier anneau concentrique.

La confrontation des données aux deux dates 1982 et 2003 met en évidence une faible évolution et la préexistence des deux gradients. La période analysée n'a enregistré qu'une légère augmentation de la proportion de surfaces d'habitat collectif, de manière globale et indépendante de la distance. À proximité immédiate des stations $(0-400 \mathrm{~m})$, les surfaces d'habitation collective ont augmenté de $17 \%$; au-delà, la croissance atteint $16 \%$. C'est surtout dans le premier anneau de $100 \mathrm{~m}$, de faible étendue, que la croissance est la plus forte : + $33 \%$. Au-delà de $100 \mathrm{~m}$, il n'existe pas à proprement parler une différenciation qui soit intrinsèque à la localisation relative au métro.

Fig. VI - surfaces d'habitation collective et individuelle, distance à la station la plus proche $(\mathbf{1 9 8 2}-2003)$ 


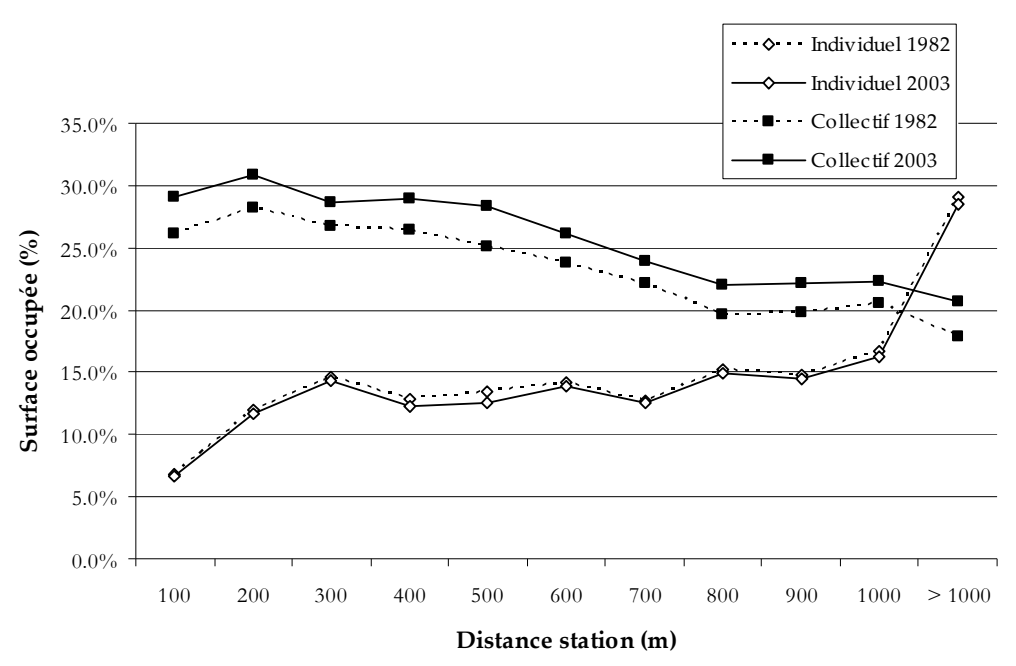

Source : RATP, IAURIF/MOS

Les surfaces d'habitat collectif de la classification en 11 modes se divisent en trois types différents dans les classifications suivantes, à 48 et 83 modes. Les surfaces d'habitat continu bas présentent un maximum de 3 étages ; les surfaces d'habitat collectif continu haut vont de 4 à 7 étages et sont typiques des centres urbains anciens ou de la période immédiatement postérieure à la Seconde Guerre Mondiale; les surfaces d'habitat collectif discontinu, de 4 étages au minimum et pouvant dépasser les 12 étages, caractérisent principalement les grands ensembles résidentiels récents (années soixante-1970 principalement). Ces types individualisés caractérisent donc des contextes urbains et des modes de consommation de l'espace différents.

Les zones d'habitat collectif continu haut se distribuent graduellement, en 2003, autour des stations du réseau métropolitain (Fig. VII). Occupant $6 \%$ de l'espace dans un rayon de 1000 mètres, ils se maintiennent autour de 3 $\%$ dans les anneaux les plus éloignés et se présentent comme le mode résidentiel le plus polarisé autour du réseau métropolitain. Quant aux surfaces d'habitat collectif discontinu, elles se structurent de manière différente, avec un maximum sur les bandes de 300 à 500 mètres, alors qu'augmente la proportion d'espace occupé par les aires d'habitation collective de faible hauteur à mesure que décroît la distance.

L'évolution qui se vérifie entre 1982 et 2003 est de faible ampleur. D'une part, la croissance des zones d'habitation discontinue est générale et statistiquement indépendante de la localisation du métro. D'autre part, l'habitat continu connaît deux tendances différentes, faiblement marquées, selon la hauteur des édifices : alors que les immeubles de 4 à 7 étages tendent à aumenter légèrement sur les bandes de 0 à 300 mètres, les immeubles plus bas, en majorité composés d'anciennes maisons en tissu resserré du début du $\mathrm{XX}^{\mathrm{e}}$ siècle, tendent à diminuer dans le premier anneau, et à se stabiliser au-delà. La conclusion s'assimile donc à celle des activités : il existe une polarisation, l'espace se stratifiant à l'échelle interne dans une quasi-sucession station de métro/habitation collective continue/zones résidentielles discontinues hautes/zones de résidences individuelles. Mais aucune altération marquante de l'usage du sol, entre 1982 et 2003, ne peut être ici envisagée.

Fig. VII - intensité résidentielle et distance à la station la plus proche. 

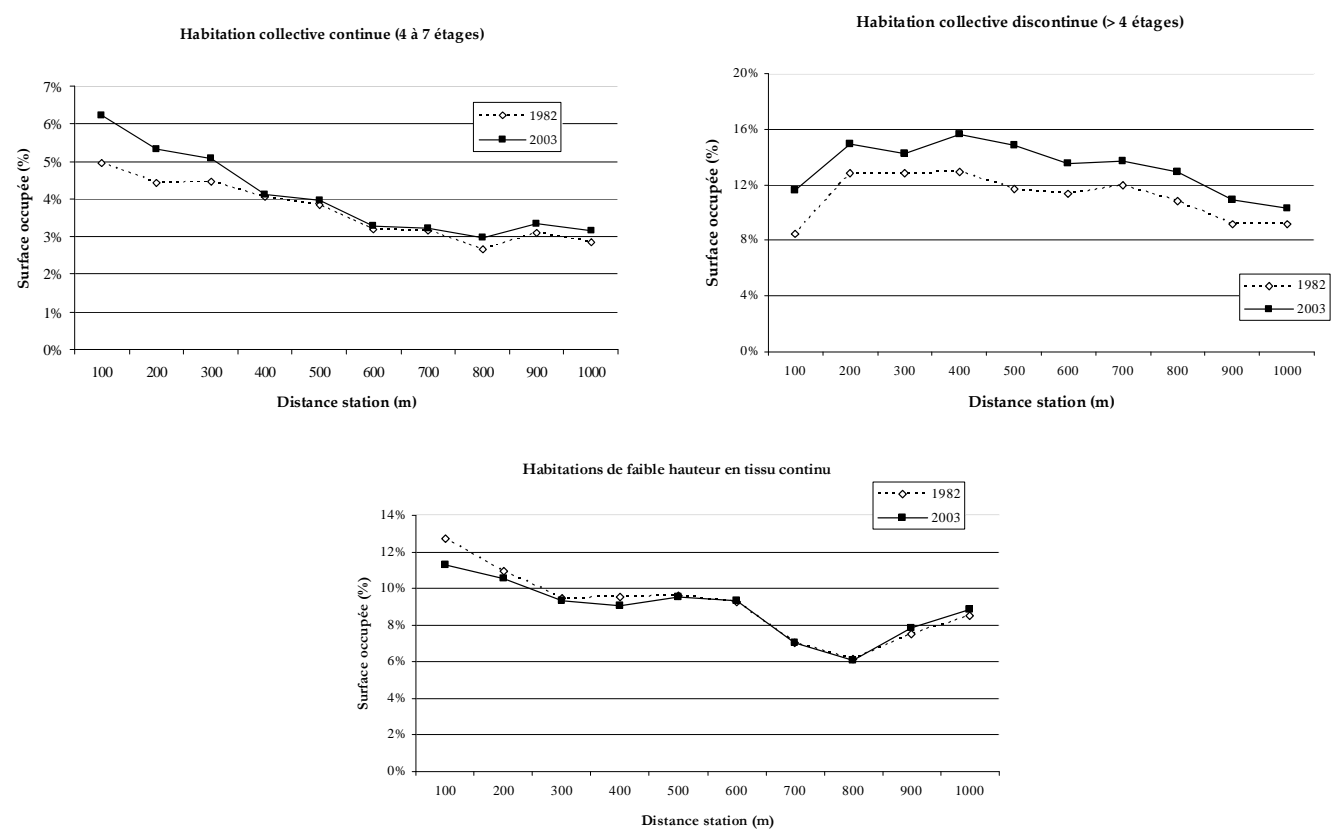

Source : IAURIF/MOS

\section{L'espace vacant urbain, un potentiel de valorisation renouvelable}

Le choix du dernier item étudié résulte de son habituelle utilisation, dans les études existantes, en tant qu'espaces potentiels de développement (IAURIF, 1999 ; Said, Santoire, Hengoat, 2001), la présence d'espaces vacants révélant des potentialités d'urbanisation et de recomposition urbaine existant sur un territoire donné. Dans cette approche classique, qui consiste à comparer 1982 et 2003 et à déduire l'évolution en hectares et en pourcentage, est habituellement évalué un solde global de la surface considérée comme «vacante ». Les chiffres montrent que, à mesure de l'éloignement du métro, la dynamique de disparition des espaces vacants diminue : dans un rayon de 500 mètres, la proportion des espaces vacants a baissé de $31 \%$; dans le deuxième rayon de 500 mètres, elle n'a diminué que de $7 \%$ et nous observons même, pour la bande 900 - 1000 mètres, que la proportion a augmenté de $23 \%$. L'interprétation générale, intuitive, est alors celle d'une meilleure valorisation des espaces libres dans les secteurs les mieux desservis.

Une approche plus dynamique, telle qu'elle apparaît sur le graphique de la Fig. III, consiste en revanche à confronter deux dynamiques contradictoires : l'apparition et la disparition simultanées de surfaces vacantes. Il s'agit, d'une part, de calculer la proportion de l'espace classé «vacant urbain » en 1982 qui devait par la suite connaître un changement d'affectation : cette part est désignée taux de modification. D'autre part est calculée la proportion de l'espace classé « vacant urbain » en 2003 et qui n'en était pas en 1982 : c'est le taux de nouveauté. La comparaison entre les deux taux permet d'approfondir les observations et de clarifier la structure de l'évolution constatée. C'est ainsi qu'entre 1982 et 2003, $88 \%$ des espaces vacants ont été transformés dans un rayon de 500 mètres autour du réseau métropolitain, correspondant à la mutation de 26,4 ha, et les chiffres décroissent une fois passée la limite de 500 mètres, avec un taux moyen de $69 \%$ d'espace vacant muté. Dans la plupart des cas, à ces surfaces se sont substitués des zones et lotissements d'activités ( $14 \%$, particulièrement au-delà de 500 mètres), des grands ensembles (11\%), des parcs et jardins, des activités en tissu urbain mixte. Cette mise en valeur de terrains non construits et inutilisés jusqu'alors a été, en revanche, partiellement compensée par l'apparition de nouveaux espaces vacants ayant pour principales origines les grandes surfaces industrielles et les activités en tissu urbain mixte. Ces nouveaux terrains vagues atteignent plus de 17 ha dans un rayon de 500 mètres et égalent presque les espaces mutés au-delà : 53 ha d'espaces vacants sont ainsi apparus entre 500 et 1000 mètres du réseau métropolitain. De cette analyse doivent ressortir trois observations principales.

Fig. VIII - mutations et nouveautés des espaces vacants (19822003). 


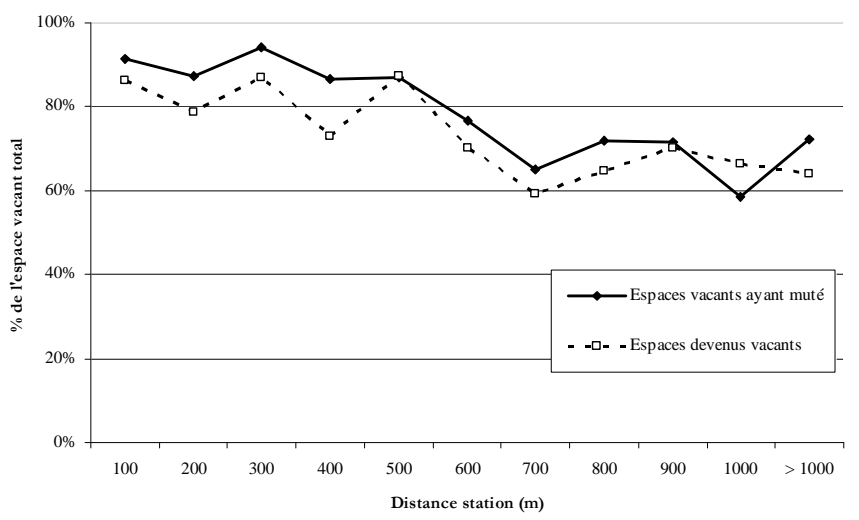

Source : IAURIF/MOS

D'abord, il existe bien une dynamique d'altération différenciée au sein de l'espace suburbain parisien qui se structure selon une distribution graduelle autour du réseau métropolitain : à moins de 500 mètres des stations du réseau, les taux de modification et de nouveauté sont supérieurs ; au-delà, ils fléchissent. Autrement dit, les modes d'usage du sol urbain central ou péricentral se différencient alors des espaces périphériques par une moindre pérennité, une plus grande instabilité, une plus grande propension à changer d'usage. Ensuite, alors qu'existent dans ces espaces péricentraux des «opportunités de recomposition» (DREIF, 1998), leur mise en valeur est partiellement compensée par l'apparition de nouveaux espaces vacants, ce qui, en termes comptables, équivaut à des modes de valorisation incomplète. Une telle neutralisation - partielle - du solde global n'est pas en soi de nature à susciter les regrets par rapport à l'action urbanistique, dans la mesure où elle témoigne de la possibilité renouvelée de recréation du tissu existant par substitution des espaces modifiables, d'une dynamique continue de transformation des usages. Il n'en demeure pas moins qu'elle affaiblit considérablement la vision d'une banlieue saturée au sein de laquelle l'espace manquerait, validant par là l'existence et le caractère essentiel de ce «front interne de recomposition » urbaine (Burgel, 1999). Enfin, l'état antérieur des nouveaux espaces vacants atteste d'une évolution pour le moins prévisible. De nature le plus souvent industrielle, qu'il s'agisse de grandes emprises d'un seul tenant ou de petits ateliers insérés dans un dense tissu résidentiel, les nouveaux espaces vacants traduisent les difficultés structurelles d'une banlieue industrielle en déshérence dont la relance tarde parfois à se mettre en route pour des questions institutionnelles, financières, mais également d'inadaptation à l'installation des réseaux (eau, électricité, assainissement), ou encore de pollution des sols. Il existe dès lors une proximité quasifonctionnelle entre des terrains anciennement industrialisés et d'autres laissés à l'abandon ou en suspens.

Les résultats restent, dans l'ensemble, mitigés. D'une part, s'il existe bien une distribution graduelle des usages selon les types d'espaces (résidentiels, économiques) et leur caractère extensif ou intensif, il reste qu'en évolution, sur une période relativement large, cet état ne varie que très peu. La pérennité des usages semble être un trait caractéristique de ces espaces que le métro dessert. Voici qui n'est pas étonnant : associés à une demande sociale ou à des opérations urbanistiques de grande ampleur, venant par conséquent compléter des structures pré-existantes, les prolongements de ligne ne se montrent guère enclins à bousculer l'ordre établi et, tout au plus, l'accompagnent timidement. Mais d'autre part, l'examen des surfaces vacantes a montré que l'instabilité de ces espaces par définition transitoires était d'autant plus aigue que le réseau métropolitain était proche. L'apparition d'une vacance, le plus souvent issue du déclin des zones industrielles de la petite couronne parisienne, reflète surtout la déconcentration rapide des emplois vers les marges urbaines. En revanche, la plus forte instabilité de ces aires libres en transition, lorsque le métro n'est pas loin, renvoie aux tensions du contrôle du sol et du marché foncier, plus prégnantes ici qu'ailleurs. C'est une ressource potentielle à prendre en considération.

\section{3 À quand l'infrastructure prescriptrice et opposable ?}

De ce jeu entre pérennité et instabilité de l'espace suburbain émerge, commune, l'incertitude quant à son devenir. Le potentiel de dynamisme existe, dont l'évolution des espaces vacants nous apporte la preuve; en revanche, le 
solde des espaces dédiés aux activités économiques et à la fonction résidentielle n'évolue pas selon les injonctions des documents régionaux. Alors que d'autres orientations, par le passé, ont cru pouvoir orienter la densification vers ces secteurs privilégiés, il devient légitime de se demander, d'une part, pourquoi la réalité n'y a pas correspondu et, d'autre part, si l'outil de planification proposé par le SDRIF dans sa version de 2007, avec ses zones de densification préférentielle, est suffisamment armé.

Il convient dans un premier temps de souligner que la part de risques prise par le décideur en matière de transports demeure peu significative. Le métro s'étant le plus souvent superposé aux structures et configurations spatiales pré-existentes, favorisant les grandes voies radiales d'accès à Paris (c'est le cas de $68 \%$ des stations suburbaines), et ce principalement pour des raisons techniques et financières, il n'a fait qu'obéir au poids historique d'un réseau-support viaire (Sander, 1997) dont les gradients repérés constituent une expression achevée. C'est en fait à une minimisation du risque financier qu'il a été procédé, en favorisant les zones où la demande s'exerçait avec une plus grande acuité, ou celles dont l'évolution était dirigée par d'autres logiques (réforme administrative de 1964 ; extension du C.B.D. de La Défense), garantissant ainsi une certaine sécurité aux gestionnaires et décideurs. La notion de rentabilité socio-économique a prévalu dans les décisions de ces prolongements de lignes : la lecture de plusieurs projets et rapports publiés au cours des années quatre-vingts et 1990 par la RATP renforce cette hypothèse. Dans le cas de la ligne 5 vers Bobigny, l'opération d'urbanisme menée à partir des années soixante-dix à la suite de la réforme administrative de 1964 a justifié la création du service (RATP, 1979b). La variante de l'extension de la ligne 7 sur le territoire de La Courneuve a été choisie de la manière suivante : " (elle) apparaitt comme une opération hautement souhaitable compte tenu du nombre d'habitants desservis, du trafic relativement important qu'il supportera et du bilan économique favorable qui en résultera » (RATP, 1975). Dans le même rapport est abandonnée l'idée de desservir le territoire de Bobigny par la ligne 11, pour des raisons techniques et financières, la rentabilité étant considérée insuffisante. Ainsi a-t-on pu estimer que jusqu'au milieu des années quatre-vingts, l'analyse coûts-avantages, en s'appuyant sur la théorie micro-économique classique, tenait le haut du pavé (Danzanvilliers, 1994). Aussi la programmation des investissements en matière de transports met-elle en jeu, dans un document de la RATP de la fin des années soixante-dix, six critères principaux : population et emplois desservis, trafic prévisible, coût de l'infrastructure par kilomètre de ligne, taux de rentabilité socio-économique, évaluation de l'intérêt vis-à-vis de l'organisation générale du réseau de transports en commun, « effet structurant sur l'urbanisme» (RATP, 1979a). Les deux derniers critères, bien que non quantitatifs, font alors l'objet d'une hiérarchisation considérée comme objective (Hugonnard, Roy, 1982).

Dans un second temps, l'absence d'alignement de certaines politiques municipales constitue un facteur de blocage. A l'échelle régionale, les modifications du gradient d'intensité de l'occupation du sol par l'habitat collectif continu haut, qui montraient une légère augmentation à moins de $500 \mathrm{~m}$ du réseau métropolitain, ne touchaient en réalité que 9 hectares ce qui, à supposer une densité équivalente à la densité moyenne de Paris, équivalait à moins de 2000 habitants. Ces modifications de faible ampleur étaient en outre portées, à $80 \%$, par à peine quatre communes: Pantin, Kremlin-Bicêtre, Clichy et Boulogne. On ne saurait mieux dire la variété de situations qu'occultent les évolutions analysées à cette échelle. Le relatif échec du SDRIF dans sa version de 1994 est dû, pour partie, au peu d'enthousiasme des édiles municipaux (Fouchier, 2007); par ailleurs, un certain nombre de possibilités de mutation ou de développement des espaces se heurtent aux orientations politiques ou urbanistiques, au manque d'adaptation des documents d'urbanisme (Said, Santoire, Hengoat, 2001): les ZAC (Zones d'Aménagement Concerté), trop peu nombreuses, trop ambitieuses ou contradictoires avec la volonté régionale, ne correspondent que rarement aux aires de desserte des infrastructures de transport; les acteurs récalcitrants souhaitent parfois conserver un habitat principalement pavillonnaire, comme à La Courneuve, se positionnent contre la densification, ainsi de la mairie des Lilas qui a procédé à la diminution du Coefficient d'Occupation du Sol (COS) dans son Plan Local d'Urbanisme, éventuellement sous la pression des habitants, favorables à une amélioration de la qualité de vie à l'échelle locale mais ne maîtrisant pas les enjeux à l'échelle globale, ainsi d'Asnières et de Gennevilliers. Cette posture se heurte aux présupposés quant aux moyens d'action des infrastructures de transport. Elle incite à se pencher sur le rôle prescriptif du transport.

Il n'est pas dans notre intention de débattre sur le niveau auquel doit s'exercer le pouvoir, ni sur les modes de partenariat que les opérations impliquent. Mais, de même que le cœur de l'agglomération est soumis aux exigences 
des riverains (Ascher, 2007), il est légitime de se poser la question de qui possède le pouvoir en matière d'urbanisme et d'aménagement du territoire, en particulier lorsque devient significative une certaine «inflation revendicative locale » (Béhar, Estèbe, 2007). Notre propos vise surtout à faire remarquer que, tant que l'élaboration d'un projet d'infrastructure de transport dont on attend des retombées en termes de localisation urbaine des acteurs (localisation résidentielle des ménages, construction de logements, de bureaux, d'activités diverses) et en termes de tissu bâti, ne donnera pas lieu à des documents d'urbanisme - au sens juridique du terme - à caractère prescriptif, opposables aux tiers, sur des aires pré-définies à un niveau supérieur, il ne faudra guère attendre que ces retombées se produisent dans la réalité.

Or, alors que le SDRIF fait une large place au principe de subsidiarité, permet une grande marge de manœuvre aux municipalités, à commencer par la définition des périmètres de densification, et y ajoute encore des zones de développement urbain non cartographiées, il ne semble pas que les couloirs préférentiels puissent devenir les valables instruments de l'urbanisme opérationnel (Amar, 2004) que la démarche intégratrice requiert. Le Conseil Économique et Social de la Région Île-de-France a d'ailleurs récemment recommandé que soient revus les niveaux de précision géographique et réglementaire des zones de densification préférentielle et conditionnelle (CESR, 2007). Sous peine de faire face au dernier mot des maires, généralement peu favorables à la densification (Renard, 2007) : la mosaïque malthusienne ajoute encore des difficultés à la fragmentation du pouvoir urbain, à l'éparpillement des compétences en matière d'urbanisme (Larroque, Margairaz, Zembri, 2002).

\section{Conclusion}

«Il y a un autre monde mais il est dans celui-ci ». Le mot est de Paul Éluard et figure dans le projet du SDRIF 2007. Une manière efficace de se représenter les possibilités de recomposition de la ville sur elle-même. Encore faut-il trouver les instruments nécessaires, mais dans quel monde sont-ils? Les différents usages du sol qui parsèment la mosaïque suburbaine présentent des rythmes d'évolution contrastés, du bref chantier aux aires résidentielles et tertiaires qui conquièrent, parcelle après parcelle, de courtes portions d'espace. Entre les deux extrêmes, une foule d'usages dont la pérennité n'est jamais garantie et dont l'évolution dépend au bout du compte de l'unique politique municipale, au nom du principe de subsidiarité ou en l'absence de contrôle de légalité. L'incursion du métro dans cet entre-deux que constitue la banlieue proche, plus que de modifier l'existant, s'est appuyée dessus ; mais, à travers l'accessibilité rapide à la capitale qu'elle permet, elle aura contribué à accroître localement la dynamique de changement lorsque les types d'occupation du sol urbain s'y prêtaient et lorsque la politique municipale l'autorisait. Il y a là, vraisemblablement, un triptyque par lequel cheminent les destins spatiaux et qui incite au dépassement des frontières.

\section{Bibliographie}

AMAR G., 2004, Mobilités urbaines : éloge de la diversité et devoir d'invention, La Tour d'Aigues : L'Aube, 251 p.

ASCHER F., 2007, «Les non-choix du SDRIF en font un catalogue, mais pas un projet stratégique », Pouvoirs Locaux, n73/II, pp. 57-60.

BECKOUCHE P., VIRE E., 1998, "La recomposition du centre économique parisien”, In Données urbaines 2, coordonné par M.F. Mattei, D. Pumain, Paris : Anthropos, coll. Economica, pp. 287-296.

BÉHAR D., ESTÈBE P., 2007, « Faut-il un gouvernement à l'̂̂le-de-France ? », Pouvoirs Locaux, n73/II, pp. 98102.

BONNAFOUS A., TABOURIN E., 1998, "Modélisation de l'évolution des densités urbaines", In Données urbaines 2, coordonné par M.F. Mattei, D. Pumain, Paris : Anthropos, coll. Economica, pp. 167-180.

BURGEL G., 1999, Paris : avenir de la France, La Tour d'Aigues : L'Aube, 198 p. 
BURGEL G., 2006, "Paris, une centralité d'exception”, In Voies publiques : espaces et pratiques de l'espace public à Paris, sous la direction de S. Texier, Paris : Ed. Picard - Ed. Pavillon de l'Arsenal, pp. 265-270.

BUTLER T., 1997, Gentrification and the Middle Classes, London : Ashgate, 188 p.

CARROUÉ L., 2000, "De tôles et de presses", in : Vallat C. (dir.), Ile-de-France : lumière des terroirs, des savoirs, des pouvoirs, Paris : Autrement, Hors-série n¹7, pp. 181-189.

CESR, 2007, « L’approbation du CESR, mais quelques réserves », Pouvoirs Locaux, n73/II.

COHEN J.-L., 2002, "Le Grand Paris, ses pôles et ses nœuds", Séminaire Conceiving "Urban Form » in the PostFordist Networked Economy, Internationales Forschungszentrum Kulturwissenschaften, Vienne, 24-25 mai 2002, Site de l'Université de Harvard ligne), <http://www.people.fas.harvard.edu/ ces/studygroups/european_city/JL_Cohen.pdf>, (dernière consultation de la page le 6 février 2006).

CORNU M., 1972, La conquête de Paris, Paris : Mercure de France, 343 p.

DREIF, 1998, Impact urbain de la réalisation en tramway de la rocade Orbitale de proche couronne : diagnostic et approche régionale. Rapport de synthèse, Paris : DREIF, Division de l'Urbanisme et du Schéma Directeur, $21 \mathrm{p}$.

FOUCHIER V., 2003, "L'étalement, la dédensification et le desserrement en Île-de-France”, In Données Urbaines 4, coordonné par M.F. Mattei, D. Pumain, Données urbaines 4, Paris : Anthropos, coll. Economica, pp. 159168.

FOURCAUT A., FLONNEAU M., 2005, Une histoire croisée de Paris et de ses banlieues à l'époque contemporaine : bilan d'étape, Paris : Mairie de Paris, 33 p.

HUGONNARD J.C., ROY B., 1982, "Le plan d'extension du metro en banlieue parisienne, un cas type d'application de l'analyse multicritère", Les cahiers scientifiques du transport, $\mathrm{n}^{\circ} 6,1 \mathrm{er}$ semestre, pp. 77 108.

IAURIF, 1999a, Etude de densification des quartiers autour des stations de transport en commun. Première phase : analyse statistique et définition typologique, Paris : IAURIF, 98 p. + annexes.

IAURIF, 2001, “L'occupation du sol en Île-de-France aujourd'hui : premier bilan du MOS 1999”, Paris : IAURIF Note rapide, $\mathrm{n}^{\circ} 282$, Mode d'occupation du sol, $\mathrm{n}^{\circ} 3,6 \mathrm{p}$.

INSEE, IAURIF, 2003, Atlas des Franciliens : activité et emplois, tome 4, Paris : INSEE, IAURIF, $121 \mathrm{p}$.

LARROQUE D., MARGAIRAZ M., ZEMBRI P., 2002, Paris et ses transports XIX ${ }^{e}-X X^{e}$ siècles : deux siècles de décisions pour la ville et sa région, Paris : Recherches, 399 p.

LÉVY J., 1994, L'espace légitime : sur la dimension géographique de la fonction politique, Paris, Presses de la Fondation Nationale des Sciences Politiques, 442 p.

MENDES L., 2006, «A nobilitação urbana no Bairro Alto: análise de um processo de recomposição sócioespacial », Finisterra, vol. XLI, n ${ }^{\circ} 1$, pp. 57-82

MERLIN P., 1997, Les transports en région parisienne, Paris : La Documentation Française, 202 p.

RAJCHMAN M., 1980, Impact du prolongement du métro à Montreuil. Etude rétrospective, Paris : IAURIF, 37 p.

RATP, 1975, Métro urbain, prolongement de la ligne 7 à la Courneuve : planches, schéma de principe, Paris : RATP, $37 \mathrm{p}$.

RATP, 1979a, Extension du métro en banlieue : évaluation globale et classement des différents prolongements, Paris : RATP - Service des Etudes Générales et du Développement, 22 p.

RATP, 1979b, Prolongement de la ligne 5 à Bobigny, Paris : RATP, 29 p. 
RENARD V., 2007, «Cycles immobiliers, problème foncier et développement urbain de l'Île-de-France », Pouvoirs Locaux, n73/II, pp. 77-82.

SDRIF, 2007, Projet arrêté par délibération du Conseil Régional le 15 février 2007, 234 p.

SAID V., SANTOIRE V., HENGOAT D., 2001, Etude de valorisation des quartiers autour des stations de transport en commun, Paris : IAURIF, 241 p.

SANDER A., 1997, "Morphogénèse des transports en commun de surface en banlieue parisienne : les occasions manquées du redéploiement", Flux, n²9, juillet-septembre, pp. 14-24.

SDAURP, 1965, Site de la DREIF, (en ligne)

<http://www.ile-de-france.equipement.gouv.fr/article.php3?id_article=970>

(dernière consultation de la page le 30 novembre 2007).

SMITH N., 1999, «À propos de yuppies et de logements : la gentrification, la restructuration sociale et le rêve urbain », Géographie, Économie et Société, vol. 1, n 1, pp. 157-196.

STP, Archives du Syndicat des Transports Parisiens, 581 WR 257, Études sur les transports (1950-1990).

WENGLENSKI S., 2003, Une mesure des disparités sociales d'accessibilité au marché de l'emploi en Île-deFrance, Thèse de doctorat : Créteil, Université de Paris XII - Val-de-Marne, Institut d'Urbanisme de Paris, $373 \mathrm{p}$. 